\title{
EFFICIENT MULTIPATH CHANNEL ESTIMATION USING A SEMI-BLIND PARAMETRIC TECHNIQUE
}

\author{
Athanasios A. Rontogiannis ${ }^{1} \quad$ Alexandra Marava $^{2}$ Kostas Berberidis $^{2} \quad$ Jacques Palicot $^{3}$ \\ ${ }^{1}$ School of Natural Resources and Enterprises Management, Univ. of Ioannina, 2 G. Seferi, 30100 Agrinio, Greece \\ ${ }^{2}$ Dept. of Computer Eng. \& Informatics / Computer Technology Institute, Univ. of Patras, 26500 Rio, Greece \\ ${ }^{3}$ INRIA/IRISA, Rennes, France \\ E-mails: arontogi@cc.uoi.gr, \{marava,berberid\}@ceid.upatras.gr,jacques.palicot@irisa.fr
}

\begin{abstract}
In this paper a parametric method for estimating the unknown channel impulse response (CIR) in a semi-blind manner is proposed. The main trait of this method is that instead of seeking the whole CIR sequence, only the unknown time delays and attenuation factors of the physical channel multipath components are estimated. The technique is based on a suitable application of the Subchannel Response Matching (SRM) criterion. The resulting cost function is separable with respect to the two sets of unknown parameters, i.e. time delays and attenuations. Thus an efficient two step optimization procedure can be applied. The new method offers significant computational savings and a lower mean square estimation error as compared to existing semi-blind channel estimation methods.
\end{abstract}

\section{INTRODUCTION}

In this paper we address the multipath channel estimation problem in wireless communication systems. Due to the multipath phenomenon in wireless applications, the introduced intersymbol interference may cause a severe degradation in a system's performance. It should be noted that the higher the symbol rate, the more the symbols spanned by the CIR. Thus, an efficient and accurate estimation of the CIR is highly desirable, in order to reduce the introduced ISI.

In many high-speed wireless applications, the propagation channel can be modeled as a specular channel consisting of a relatively small number of dominant rays [1], each one being characterized by its time delay and attenuation factor. Then, provided that the transmitter and receiver filters are known, the channel estimation task is reduced to that of estimating the parameters of the multipath channel components. Two are the main advantages of using such a parametric approach. First, the number of required data is reduced, resulting in a significant saving in complexity. Second, the mean square channel estimation error is expected to be lower since the number of parameters to be estimated is smaller compared to non-parametric channel estimation [4].

In a wireless communication channel, bandwidth is a precious resource, therefore the need for training sequence reduction is imperative. Thus, conventional training based channel estimation methods are inappropriate especially when the channel span is large as in high rate applications. In such cases semiblind estimation techniques may be employed. The main trait of a semiblind scheme is that a purely blind criterion is suitably modified so as to incorporate

This work was supported by Computer Technology Institute (CTI) and France Telecom. information from a short training sequence, [2]. The performance of a semiblind method, if properly designed, can be significantly improved against the corresponding blind method.

In this paper we propose a novel semi-blind technique, which exploits the specular channel structure. By applying the well-known SRM criterion [3] to the problem at hand, we end up with a least squares (LS) problem, which is separable with respect to the unknown parameters, i.e. time delays and attenuation factors. It is then shown that the optimization problem can be separated to two different sub-problems. A sub-problem which is non-linear with respect to the time delays and a sub-problem which is linear with respect to the attenuation parameters. After revealing the special structure of the non-linear problem, a computationally efficient linear search method for the estimation of the unknown time delays is developed. In the sequel, the Gauss-Newton algorithm may be applied in order to further improve the accuracy of the estimated values. Finally, the attenuation parameters are estimated by solving a linear LS problem. The new method is very simple to implement and for the same degree of estimation accuracy has a computational complexity which is much lower as compared to other related channel parameter estimation methods [4]. Moreover, it offers the possibility of trading off performance to complexity in an easy manner. The proposed method yields good estimates even in cases of closely spaced time delays. The performance of the new method has been justified theoretically and tested through extensive simulations.

The paper is organized as follows. In Section 2 the multipath channel model is defined and the problem is formulated. In Section 3 the new method is derived and in Section 4 simulation results verifying the performance of the new method are provided.

\section{PROBLEM FORMULATION}

\subsection{Channel Model}

In general the CIR encountered in wireless communication systems has a form which varies significantly depending on several factors. However a common trait in most cases, particularly in high speed applications, is that the multipath channel tends to be of a discrete form, i.e. it consists of a number of dominant multipath components. More specifically, if the CIR is assumed to be time invariant within a small-scale time interval then it may be written as

$$
h_{c}(t)=\sum_{k=0}^{p-1} a_{k} \delta\left(t-\tau_{k}\right)
$$

where $a_{k}$ and $\tau_{k}$ are the complex attenuation factor and the delay, respectively, of the $k-t h$ multipath component. Without loss 
of generality, it is assumed that $\tau_{0}<\tau_{1}<\ldots<\tau_{p-1}$. Thus the problem of multipath CIR estimation is reduced to the smaller problem of delay and complex attenuation parameters estimation.

Let $g(t)$ be the pulse shape filter (convolution of transmitter and receiver filters). In this paper, it is assumed that $g(t)$ is a raised cosine function and has finite support, i.e. $g(t)=0$ for $t \notin\left[0,2 L_{g} T\right]$, where $T$ is the symbol period. The overall impulse response $h(t)$ of the communication system is then given as the convolution of $h_{c}(t)$ with $g(t)$. Furthermore, we consider the multichannel model, in which the channel output is oversampled by a factor of $N_{s}$ samples per symbol period. For simplicity we take $N_{s}=2$. Thus the sampled overall CIR is expressed by two vectors, one for each subchannel, i.e. for $i=1,2$

$$
\boldsymbol{h}_{i}^{T}=\left[h\left(\frac{(i-1)}{2} T\right) h\left(T+\frac{(i-1)}{2} T\right) \ldots h\left(L T+\frac{(i-1)}{2} T\right)\right]
$$

where $L T$ is the span of the overall CIR. It is straightforward that the subchannels' impulse responses can be written in the form

$$
\boldsymbol{h}_{i}=\boldsymbol{G}_{i}(\boldsymbol{\tau}) \mathbf{a}, \quad i=1,2
$$

where $\boldsymbol{\tau}=\left[\begin{array}{llll}\tau_{0} & \tau_{1} & \ldots & \tau_{p-1}\end{array}\right]^{T}$ and $\mathbf{a}=\left[\begin{array}{llll}a_{0} & a_{1} & \ldots & a_{p-1}\end{array}\right]^{T}$. Finally $\boldsymbol{G}_{i}(\boldsymbol{\tau})$ is an $(L+1) \times p$ matrix whose columns are delayed versions of $g(t)$ depending on the unknown parameters $\tau_{k}$. Specifically this matrix has the form

$$
\begin{gathered}
\boldsymbol{G}_{i}(\boldsymbol{\tau})=\left[\boldsymbol{g}_{i}\left(\tau_{0}\right) \boldsymbol{g}_{i}\left(\tau_{1}\right) \ldots \boldsymbol{g}_{i}\left(\tau_{p-1}\right)\right] \\
\text { with } \boldsymbol{g}_{i}\left(\tau_{k}\right)=\left[g\left(\frac{i-1}{2} T-\tau_{k}\right) \ldots g\left(L T+\frac{i-1}{2} T-\tau_{k}\right)\right]^{T} .
\end{gathered}
$$

\subsection{The Semi-blind Parametric Cost Function}

The goal of the new parametric method is the estimation of the unknown parameters' vectors $\boldsymbol{\tau}$ and $\mathbf{a}$, using a small number of information symbols which are assumed to be known at the receiver. The starting point for the derivation of the new algorithm is the SRM concept, which wisely exploits the multichannel structure [3]. More specifically, in the two channels, noise-free case, the following relation holds

$$
\left\{y_{1}\right\} \star\left\{h_{2}\right\}=\left\{y_{2}\right\} \star\left\{h_{1}\right\}
$$

where $\star$ stands for convolution and $\left\{y_{i}\right\}, i=1,2$, denotes the output sequence of subchannel $i$. Moreover, the subchannels' output samples can be written as

$$
y_{i}(n)=\mathbf{h}_{i}^{T} \mathbf{s}_{L}(n)+w_{i}(n), \quad i=1,2
$$

where $\mathbf{s}_{L}^{T}(n)=[s(n), s(n-1), \ldots, s(n-L)]$ is the common input data vector and $w_{i}(n)$ stands for the output noise of subchannel $i$ at time $n$. The input sequence is assumed to be i.i.d. and independent of the noise sequences.

Let us assume that the output samples $y_{i}(k), \ldots, y_{i}(k+N-1)$, $i=1,2, N>L$ as well as the input symbols $s(k-L), \ldots, s(k+$ $M)$ are available at the receiver. $M$ is of the order of $L$ and $M \ll$ $N$. Then in the absence of noise and using (3) and (4) we have the following system

$$
\left[\begin{array}{cc}
Y_{2} & -Y_{1} \\
S_{M L} & \mathbf{0} \\
\mathbf{0} & S_{M L}
\end{array}\right]\left[\begin{array}{l}
\mathbf{h}_{1} \\
\mathbf{h}_{2}
\end{array}\right]=\left[\begin{array}{c}
\mathbf{0} \\
\mathbf{y}_{1 M} \\
\mathbf{y}_{2 M}
\end{array}\right]
$$

where

$$
Y_{i}=\left[\begin{array}{ccc}
y_{i}(L+k) & \cdots & y_{i}(k) \\
y_{i}(L+k+1) & \cdots & y_{i}(k+1) \\
\vdots & \vdots & \vdots \\
y_{i}(k+N-1) & \cdots & y_{i}(k+N-L-1)
\end{array}\right]
$$

$$
S_{M L}=\left[\begin{array}{ccc}
s(k) & \cdots & s(k-L) \\
s(k+1) & \cdots & s(k+1-L) \\
\vdots & \vdots & \vdots \\
s(k+M) & \cdots & s(k+M-L)
\end{array}\right]
$$

and $\mathbf{y}_{i M}^{T}=\left[y_{i}(k), y_{i}(k+1), \ldots, y_{i}(k+M)\right], i=1,2$.

In (5), the first set of equations implements the SRM criterion, as expressed by (3) and the other two sets of equations incorporate the known symbols according to (4). By imposing the channel parametric structure, (5) is written as

$$
Y_{S} G(\boldsymbol{\tau}) \mathbf{a}=\mathbf{z}
$$

where

$$
Y_{S}=\left[\begin{array}{cc}
Y_{2} & -Y_{1} \\
S_{M L} & \mathbf{0} \\
\mathbf{0} & S_{M L}
\end{array}\right], \quad \mathbf{z}=\left[\begin{array}{c}
\mathbf{0} \\
\mathbf{y}_{1 M} \\
\mathbf{y}_{2 M}
\end{array}\right], \quad G(\boldsymbol{\tau})=\left[\begin{array}{l}
G_{1}(\boldsymbol{\tau}) \\
G_{2}(\boldsymbol{\tau})
\end{array}\right]
$$

When the channel is corrupted by noise, we can estimate the channel parameters $\mathbf{a}, \boldsymbol{\tau}$ by solving the following LS problem

$$
\min _{\mathbf{a}, \boldsymbol{\tau}}\|\mathbf{z}-\Phi(\boldsymbol{\tau}) \mathbf{a}\|^{2}, \quad \Phi(\boldsymbol{\tau})=Y_{S} G(\boldsymbol{\tau})
$$

In the next section the form of the non-linear cost function of (9) will be explored, in order to reveal and take advantage of its possible salient characteristics.

\section{DERIVATION OF THE ALGORITHM}

The proposed efficient solution is based on two main results. First, it is shown that the optimization problem can be split up with respect to the delay and the attenuation parameters. Second, the delays are shown to be decoupled between each other allowing for their efficient estimation.

\subsection{Separable form of the problem}

It is readily seen that the non-linear LS problem in (9) is separable with respect to the unknown parameters $\boldsymbol{\tau}$ and $\mathbf{a}$. In particular, the LS cost function is nonlinear with respect to the delays $\boldsymbol{\tau}$ and linear with respect to the attenuation factors a. For this type of cost functions the optimization process can be conducted separately with respect to the distinct parameter sets $\boldsymbol{\tau}$ and $\mathbf{a}$, [5]. More specifically

- The delay parameters $\tau$ are obtained from the solution of the following non-linear optimization problem

$$
\boldsymbol{\tau}_{\text {opt }}=\arg \min _{\boldsymbol{\tau}}\{f(\boldsymbol{\tau})\}
$$

with

$$
f(\boldsymbol{\tau})=\left\|\left(I-\Phi(\boldsymbol{\tau}) \Phi^{\dagger}(\boldsymbol{\tau})\right) \mathbf{z}\right\|^{2}
$$

- The attenuation parameters a are determined by the linear LS method as

$$
\mathbf{a}_{o p t}=\Phi^{\dagger}\left(\boldsymbol{\tau}_{o p t}\right) \mathbf{z}
$$

where $\dagger$ denotes the pseudoinverse of a matrix. Note from (10) and (11) that $\boldsymbol{\tau}_{\text {opt }}$ is the value of the delay vector which minimizes the projection of vector $\mathbf{z}$ to the orthogonal complement of the space spanned by the columns of $\Phi(\boldsymbol{\tau})$. Consequently the same vector maximizes the projection of $\mathbf{z}$ to the column space of $\Phi(\boldsymbol{\tau})$ and the optimization problem can be written in the following equivalent form

$$
\boldsymbol{\tau}_{\text {opt }}=\arg \max _{\boldsymbol{\tau}}\{\boldsymbol{F}(\boldsymbol{\tau})\}, \quad F(\boldsymbol{\tau})=\left\|\Phi(\boldsymbol{\tau}) \Phi^{\dagger}(\boldsymbol{\tau}) \mathbf{z}\right\|^{2}
$$


The non-linear cost function in (13) can be treated either by performing a multidimensional search in the space of parameter set $\boldsymbol{\tau}$ or by applying a non-linear optimization search method, e.g. a Newton type method. In the former case the computational burden may be prohibitive, in the latter the procedure may be trapped in a local minimum, away from the global solution. In the following section, we exploit the special form of the cost function $F(\cdot)$ to derive an efficient and accurate method for estimating $\boldsymbol{\tau}_{o p t}$.

\subsection{Decoupling with respect to delays}

Let us start with an example and assume that the multipath channel contains two unknown delays, namely $\tau_{0}$ and $\tau_{1}$. In Fig.1, the function $f(\boldsymbol{\tau})$ is plotted versus $\tau_{0}, \tau_{1}$. By inspecting the plotted function we realize that instead of performing a 2-dimensional search, two 1-dimensional searches are adequate for the location of the global minimum. More specifically, one can initially fix the value of $\tau_{1}$ and then minimize the cost function by varying parameter $\tau_{0}$. In the sequel, the same procedure can be repeated for parameter $\tau_{1}$ after fixing the value of $\tau_{0}$ to the optimum value obtained from the first search. Note that, as expected, function $F(\cdot)$ has a similar form and a similar approach can be followed.

In the lines to follow, we generalize the concept described above by exploiting the special form of function $F(\cdot)$ and showing that under certain circumstances, which are satisfied in the applications of interest, the cost function can be decoupled with respect to the delay parameters. Indeed, since $\Phi(\boldsymbol{\tau}) \Phi^{\dagger}(\boldsymbol{\tau})$ is a projection operator, the cost function $F(\cdot)$ is expressed as follows

$$
F(\boldsymbol{\tau})=\mathbf{z}^{H} \Phi(\boldsymbol{\tau})\left(\Phi^{H}(\boldsymbol{\tau}) \Phi(\boldsymbol{\tau})\right)^{-1} \Phi^{H}(\boldsymbol{\tau}) \mathbf{z}
$$

By setting $\mathbf{q}(\boldsymbol{\tau})=\Phi^{H}(\boldsymbol{\tau}) \mathbf{z},(14)$ is written as

$$
F(\boldsymbol{\tau})=\mathbf{q}^{H}(\boldsymbol{\tau}) A^{-1}(\boldsymbol{\tau}) \mathbf{q}(\boldsymbol{\tau})
$$

with

$$
\begin{gathered}
A(\boldsymbol{\tau})=G^{H}(\boldsymbol{\tau}) Y_{S}^{H} Y_{S} G(\boldsymbol{\tau}) \\
\mathbf{q}^{H}(\boldsymbol{\tau})=\left[\begin{array}{llll}
q\left(\tau_{0}\right) & q\left(\tau_{1}\right) & \cdots & q\left(\tau_{p-1}\right)
\end{array}\right]
\end{gathered}
$$

and $q\left(\tau_{i}\right)=\mathbf{z}^{H} Y_{S} \mathbf{g}\left(\tau_{i}\right)$. That is, the $i$-th element of vector $\mathbf{q}$ is a function of the $(i-1)$-th delay parameter only, for $i=1,2, \ldots, p$. Due to the form of vector $\mathbf{q}$, we deduce from (15) that $F(\boldsymbol{\tau})$ would be decoupled with respect to the delay parameters, if matrix $A^{-1}(\boldsymbol{\tau})$ was diagonal and its $i$-th diagonal element was a function of $\tau_{i-1}$ only. Although such an ideal case is not valid in general, it can be shown [6] that under reasonable assumptions $A^{-1}(\boldsymbol{\tau})$ is diagonally dominant. As a result, cost function $F(\boldsymbol{\tau})$ can be approximated by a summation of $p$ positive terms, each of which is a function of one delay parameter only. Therefore, the optimization search can be performed separately for each $\tau_{i}, i=0,1, \ldots, p-1$ and independently of the other delay parameters. In other words, instead of a $p$-dimensional search of exponential complexity, $p$ linear searches are sufficient for the solution of the optimization problem, which dramatically reduces complexity.

\subsection{Solution refinement using Gauss-Newton}

The solution obtained from the procedure described above, say $\boldsymbol{\tau}^{(0)}$, is close to $\boldsymbol{\tau}_{\text {opt }}$ and can be further improved by applying a few steps of a Gauss-Newton (G-N) search method, expressed by the following update formula

$$
\boldsymbol{\tau}^{(n+1)}=\boldsymbol{\tau}^{(n)}-\mu H^{-1}\left(\boldsymbol{\tau}^{(n)}\right) V\left(\boldsymbol{\tau}^{(n)}\right), \quad n=0,1, \ldots
$$

where $\mu$ is the step size parameter of the G-N search and $V(\cdot), H(\cdot)$ stand for the gradient vector and the Hessian matrix of $F(\cdot)$ respec- tively. It can be shown that these quantities can be expressed as follows

$$
\begin{gathered}
V(\boldsymbol{\tau})=E^{H}(\mathbf{a}, \boldsymbol{\tau}) E_{\boldsymbol{\tau}} \\
R(\boldsymbol{\tau})=E_{\boldsymbol{\tau}}^{H}\left(I-E_{\mathbf{a}}\left(E_{\mathbf{a}}^{H} E_{\mathbf{a}}\right)^{-1} E_{\mathbf{a}}^{H}\right) E_{\boldsymbol{\tau}}
\end{gathered}
$$

where $E(\mathbf{a}, \boldsymbol{\tau})=\mathbf{z}-\Phi(\boldsymbol{\tau}) \mathbf{a}$ is the error vector and $E_{\mathbf{a}}=\frac{d E(\mathbf{a}, \boldsymbol{\tau})}{d \mathbf{a}}=-\Phi(\boldsymbol{\tau}), E \boldsymbol{\tau}=\frac{d E(\mathbf{a}, \boldsymbol{\tau})}{d \boldsymbol{\tau}}=-Y_{S} \frac{d G(\boldsymbol{\tau})}{d \boldsymbol{\tau}}(21)$ The vector of attenuation parameters is recomputed in each step of the G-N search as $\mathbf{a}^{(n)}=\Phi^{\dagger}\left(\boldsymbol{\tau}^{(n)}\right) \mathbf{z}$. The G-N search is guaranteed to reach the global optimum point, under the assumption that $\boldsymbol{\tau}^{(0)}$ is in the neighborhood of this point. As a result, the estimation accuracy is improved after running a few steps of the G-N algorithm.

\section{Summary of the method}

In order to apply the new estimation method, a $p$-dimensional grid must be initially defined with a linear step size say $\delta$. Then, $F(\boldsymbol{\tau})$ is evaluated in certain points of this grid according to the procedure described in section 3.2. The basic steps of the proposed method are summarized below.

1. Set values $\hat{L}, \hat{p}$ for unknown $L, p$, respectively.

2. Initialize $\tau_{i}, i=0,1, \ldots, p-1$ with distinct random values in the interval $[0, L T]$.

3. Choose a linear search step size, $\delta$ and set $i=1$.

4. Minimize $F(\boldsymbol{\tau})$ with respect to $\tau_{i}$. Find $\tau_{i, \text { opt }} \equiv \tau_{i}^{(0)}$ by evaluating the function at $\tau_{i}=j \delta, \quad j=0,1, \ldots, \frac{\hat{L} T}{\delta}$

5. Set $\tau_{i}^{(0)}, i=i+1$ and repeat from step 3 until $i=\hat{p}$.

6. Run a Gauss-Newton search in the neighborhood of $\boldsymbol{\tau}^{(0)}$ to improve the estimation accuracy.

7. Obtain the attenuation parameters from (12).

Note that there is a trade-off between the number of evaluation points, which is a function of $\delta$ and the number of iterations of the G-N method. As the size of $\delta$ decreases, the estimation accuracy of the proposed linear search method is improved and a lower number of G-N steps is required. As shown in the simulations, the performance of the new method is not affected by overmodeling of the channel length and/or the number of multipath components. Due to space limitations a theoretical proof of this property of the proposed method is omitted.

\section{SIMULATION RESULTS}

The performance of the new method was tested via extensive simulations. In our experiments, the input sequence was taken from a QAM-16 alphabet and passed through different multipath channels. At the channel output, AWGN was added to yield an SNR varying between 16 and $26 \mathrm{~dB}$. The new method was compared with the non-parametric semi-blind SRM-based method presented in [2], in which the CIR is estimated in a non-parametric manner, using a linear combination of a blind and a non-blind cost function. For each experiment the data sequence consisted of 200 samples. 
In Fig. 2 the results for a medium length channel, with parameter vectors $\boldsymbol{\tau}=\left[\begin{array}{llll}0 & 2.9 & 7.3 & 13.7\end{array}\right]$ and $\mathbf{a}=\left[\begin{array}{lll}0.9+0.1 j & -0.4-\end{array}\right.$ $0.3 j 0.0+0.2 j 0.2-0.1 j]$, are depicted. The pulse shape filter was a raised cosine filter with a roll-off factor equal to 0.3 and $L_{g}=3$, i.e. the total channel span was $L=20$. A number of 30 samples was used for training and the linear search step size was set to 0.1 . The two methods were compared using the root-mean-square-error between actual and estimated CIRs, i.e.

$$
R M S E=\sqrt{(1 / Q) \sum_{k=1}^{Q} \sum_{i=1}^{2 L}\left(h_{a c t}(i)-h_{e s t}^{k}(i)\right)^{2}}
$$

where $Q$ is the number of independent experiments, $h_{a c t}(i)$ is the $i$-th element of the actual $T / 2$-spaced CIR and $h_{e s t}^{k}(i)$ is the $i$-th element of the estimated CIR during the $k$-th simulation. In our experiments, $Q=100$ and both CIR sequences $\left\{h_{a c t}\right\}$ and $\left\{h_{e s t}^{k}\right\}$ are normalized to have unity norm. In Fig. 2 the RMSE of the new parametric semi-blind channel estimation method is compared to the RMSE of the algorithm proposed in [2]. The RMSE of the new method is also plotted in case of overmodeling in $p$. The new parametric method outperforms significantly the non-parametric semiblind SRM method for all SNRs considered. Moreover, in case of overmodeling in $p$, the performance remains unaffected. Note that the performance of the new method could have been further improved by applying a few steps of the G-N method to get more accurate estimates of the delays.

Finally, the performance of the two methods in case $L$ is overestimated, is illustrated in Fig. 3. The SNR was set to $20 \mathrm{~dB}$. It is obvious that the new method is quite robust in channel order overestimation, in contrast to the non-parametric semiblind SRM whose performance deteriorates significantly as the degree of overmodeling increases.

\section{CONCLUSION}

A new efficient semi-blind technique for estimating the time delays and attenuation factors of a multipath channel has been developed. The efficiency of the technique, which is based on the SRM criterion, is due to a proper exploitation of the particular properties of the minimized cost function. The new method is very simple to implement and for the same degree of accuracy has a computational complexity which is much lower as compared to the existing semiblind CIR estimation methods. Extensive simulation results have confirmed the theoretically expected performance of the method.

\section{REFERENCES}

[1] T.S. Rappaport, "Wireless Communications Principles and Practice", Prentice-Hall, 1996.

[2] J.L. Babat, S.V. Schell, "Channel Identification Using a Combination of Blind and Nonblind Methods", SPIE, vol. 2605, 1995, pp. 202-211.

[3] G. Xu, H. Liu, L. Tong, T. Kailath, "A least-squares approach to blind channel identification", IEEE Trans. on Signal Proc., vol. 43, No. 12, Dec. 1995, pp. 2982-93.

[4] L. Perros-Meilhac, E. Moulines, K. Abed-Meraim, P. Chevalier, P. Duhamel, "Blind Identification of Multipath Channels: A Parametric Subspace approach", IEEE Trans. on Signal Proc., July 2001, pp. 1468-80.
[5] A. Bjorck, "Numerical methods for least squares problems", Soc. for Ind. and Applied Math. (SIAM), 1996.

[6] A.A. Rontogiannis, A. Marava, K. Berberidis, and J. Palicot, "Semi-Blind Estimation of Multipath Channel Parameters Via a Separable Least Squares Technique", in Proc. of the 14th DSP Conference, July 2002.

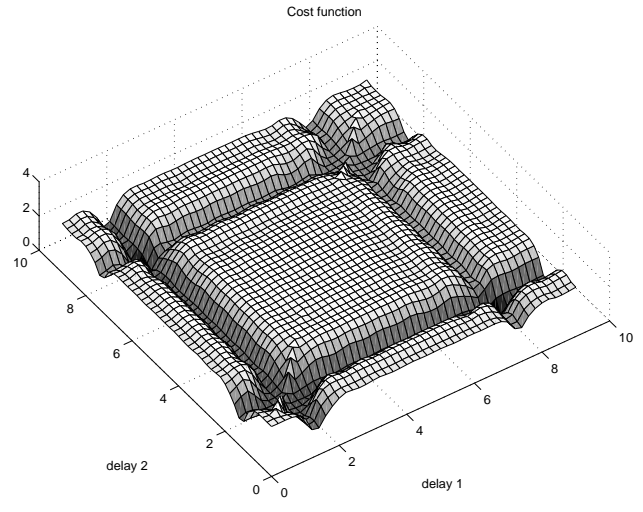

Fig. 1. Cost function, $\boldsymbol{\tau}=\left[\begin{array}{ll}1.2 & 7.4\end{array}\right]^{T}$ and $\boldsymbol{a}=\left[\begin{array}{ll}0.5 & 0.5\end{array}\right]^{T}$

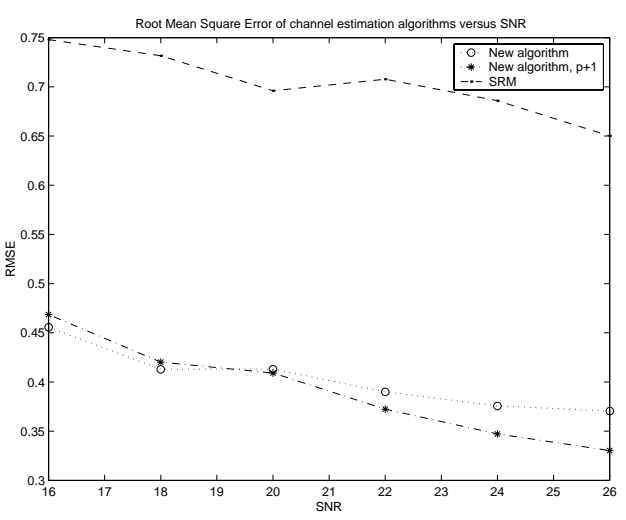

Fig. 2. RMSE curves for different SNRs

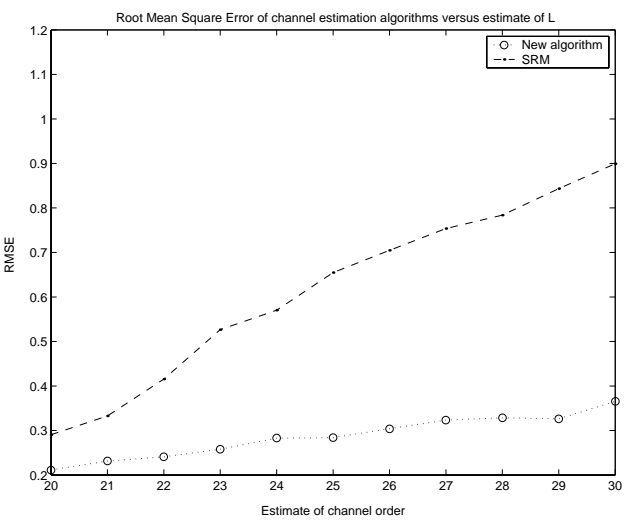

Fig. 3. RMSE curves in case of channel order overestimation 